Treatments of contextual effects in the social science literature have traditionally focused on statistical phenomena more than on social processes. Typically, the existence of contextual processes has been inferred on the basis of "group-level" effects (as contrasted with "individual-level" effects). This article seeks to redress that imbalance by focusing on underlying processes through which social structure and social interaction may impinge upon individuals. Four alternative generating mechanisms for contextual effects are discussed, along with their implications for model specification and estimation; only one of these, however, is found to be compatible with the notion of modeling individual outcomes as a result of group processes (endogenous feedback). Methods for consistent parameter estimation in endogenous feedback models are presented, based on maximum likelihood estimators for models with lattice autoregressive structure and their extension to models with latice autocorrelated error structures. Moreover, since the latter often arise by failure to specify contextual processes explicitly, conventional statistical definitions of contextual effects are shown to be confounded with the resulting specification bias.

\title{
INDIVIDUALS AND SOCIAL STRUCTURE
}

\section{Contextual Effects as Endogenous Feedback}

\author{
LUTZ ERBRING \\ University of Michigan
}

ALICE A. YOUNG

Carnegie-Mellon University

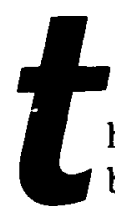

he impact of social structure on individual behavior has been a recurrent theme of social theory and research, from Durkheim's discussion of "social facts" onward. The sociological literature is rich in well-meaning exhortations to "bring society back in" (Barton, 1968)—especially since the advent of the sample survey. Coleman (1958) notes the pitfalls of survey research that treats individuals as though existing outside any context of social interaction. Blau (1960) points out the importance of structural

\footnotetext{
AUTHORS' NOTE: An earlier version of this paper was presented at the 1977 Annual Meeting of the American Sociological Association, Chicago, Illinois. We are grateful to David Jackson for many helpful suggestions, and to Duane Alwin, Arthur Goldberger, and Neil Henry for comments on a previous draft.
}

SOCIOLOGICAL METHODS \& RESEARCH, Vol. 7 No. 4, May 1979 396-430 (C) 1979 Sage Publications, Inc. 
effects that link the distribution of personal attributes within a group to individual performance. Lazarsfeld and Menzel (1969), Davis et al. (1961), and Boudon (1967) have provided typologies of a variety of group-level effects on individual outcomes. Yet the fundamental issue of how to model the process(es) underlying social context effects has remained unresolved.

While most social scientists would probably endorse programmatic declarations about the importance of social structure, the issue becomes more difficult as soon as one presses for a specific definition of what, exactly, is meant by "social structure." Is it the effects of social interaction on individuals' beliefs, expectations, and behaviors as in the study of conformity, contagion, diffusion, or influence processes? Is it the particular pattern of social relationships that in turn constrains, channels, or otherwise determines the distribution of opportunities for such interactions? Is it the specific content of transactions which take place among interacting individuals that determines the effects of social structure? Must the operational concept of social structure itself be made contingent on the particular types of social interaction that are assumed to affect individual outcomes? Unfortunately even those who advocate the theoretical importance of social context tend to leave the meaning of their message somewhat ambiguous.

The case becomes doubly confounded in conjunction with the empirical problems of separating the effects of individual attributes from the effects of social context in the determination of individual outcomes. Here the potentially rich notion of social structure tends to become diluted into mere aggregations of individual attributes or into group-level properties in a global sense. Most assessments of "contextual effects" along these lines are represented by alternative versions of linear models in which measures of individual outcomes are regressed on combinations of individual attributes and their group-level averages. In brief, what is left of group "structure" is a simple measure of group composition: a mean score. Moreover, such studies fail to provide a consistent rationale for substantive mechanisms that might connect individual outcomes even with group composition, let alone group structure. 
A number of lingering controversies have plagued this traditional formulation of "contextual effects." Thus, Hauser (1970a, $1970 \mathrm{~b}, 1974)$ rejects the use of group averages alongside individual attributes as by-products of model underspecification at the individual level and as statistical artifacts devoid of a corresponding substantive process. Alwin (1976) has further clarified the algebraic relationships between several alternative specifications of this type which have commonly been employed to estimate contextual effects. Campbell and Alexander (1965) and Alexander and McDill (1976) argue that the effects of group structure on individual outcomes are mediated through interaction with "significant others" and thus should be represented by the mean values of the attributes of relevant peers rather than of the entire group. Finally, Hannan et al. (1976) demonstrate the importance of the choice of level of analysis and of the inclusion of all relevant independent variables in the analysis.

In this article we focus on the nature of intervening mechanisms that mediate the effect of social structure on individual outcomes. We attempt to show that this focus is helpful in making the necessary transition from estimating contextual effects to providing contextual explanations. We shall illustrate our argument with alternative explanations that have been discussed in the study of school effects, though our conclusions are applicable to a large variety of substantive problems involving contextual effects.

A careful examination of alternative mechanisms through which school context effects on individual aspirations or achievements might come about reveals that the specifications usually employed to model these effects are inaccurate representations of the manner in which group structure may affect individual outcomes. We then propose a class of endogenous feedback models that do provide an adequate formalization of contextual effects and permit, indeed require, the inclusion of social structure as an explicit part of model specification. We also discuss some special estimation problems raised by this new model and present several approaches for dealing with them. 


\section{AN ANALYSIS OF CONTEXTUAL MECHANISMS}

If social context is to affect individual behavior, such effects must be mediated through processes that are somehow contingent upon the social structure in which the individual is embedded. Thus, a specification of group-level effects on individual outcomes requires, first of all, a careful examination of possible linkage mechanisms through which the hypothesized "contextual effects" might operate. In particular, we shall argue that contextual effects must be conceptualized as a consequence of processes of interaction among individuals in a social network. Interaction may involve either actual face-to-face contact between pairs of individuals or symbolic categorical relations shared among all individuals in a given group. Face-to-face interaction would give rise to social processes of contagion (diffusion, persuasion) underlying such group phenomena as assimilation (conformity, consensus, "pull") and contrast (polarization, differentiation, "push"). Symbolic interaction would give rise to socialpsychological processes of comparison underlying such "reference group" phenomena as competition, emulation, identification, facilitation and inhibition. Without reference to social interaction in either form, the notion of contextual effects tends to become theoretically vacuous.

The traditional treatment of contextual effects has proceeded from a statistical formalization based on "individual-effect-plusgroup-effect" to the imputation of alternative contextual mechanisms purporting to explain or account for statistical results. In this section we shall pursue a different strategy by examining the relationship between formal specifications and substantive mechanisms from two perspectives: (1) treating the conventional statistical model of contextual effects as a structural specification, what substantive mechanisms are implied? (2) treating alternative conventional interpretations of contextual effects as substantive mechanisms, what structural specifications are implied, and how are they related to the traditional formulation? 


\section{CONVENTIONAL FORMULATION: SOCIAL TELEPATHY}

Stripped to its bare bones, the standard contextual effects model can be represented by the following structural equation:

$$
y_{1 j}=a+b_{1} x_{1 j}+b_{2} \bar{x}_{j}+e_{i j}
$$

where $y_{i j}$ might represent the academic achievement of the $i^{\text {th }}$ student in the $j^{\text {th }}$ class, $x_{i j}$ might be a measure of intellectual ability for the same student, and $\bar{x}_{j}$ would be the average value of student ability in the $\mathrm{j}^{\text {th }}$ class; $\mathrm{e}_{\mathrm{ij}}$ is an error term representing all unspecified causes of $y_{i j}$. The coefficients $a, b_{1}$, and $b_{2}$ can be estimated by ordinary least squares regression if the usual OLS assumptions about the error terms can be justified (i.e., the $e_{i j}$ have zero expected value, are independent and homoskedastic across all values of $i$ and $j$, and are independent of $x_{i j}$ and of $\bar{x}_{j}$ ).

In order to highlight the implications of the model specified in equation 1 , let us first write the average ability score, $\overline{\mathbf{x}}_{\mathrm{J}}$, explicitly as the mean of individual student scores, i.e.,

$$
y_{i j}=a+b_{1} x_{i j}+\left(b_{2} / n_{j}\right)\left[x_{1 j}+\ldots+x_{i j}+\ldots+x_{\left.n_{j}\right]}\right]+e_{i j}
$$

where $n_{j}$ is the number of students in the $j^{\text {th }}$ class. By collecting terms for the $\mathrm{i}^{\text {th }}$ student and for all other $\left(\mathrm{i}^{\text {th }}\right)$ students in the $\mathrm{j}^{\text {th }}$ class, equation 2 becomes

$$
y_{i j}=a+\left(b_{1}+b_{2} / n_{j}\right) x_{i j}+\left(b_{2} / n_{j}\right) \sum_{i^{\prime} \neq i} x_{i^{\prime} j}+e_{i j}
$$

or

$$
y_{i j}=a+b_{1}^{*} x_{i j}+b_{2} \bar{x}_{i^{\prime} j}+e_{i j}
$$

where

$$
b_{1}^{*}=\left(b_{1}+b_{2} / n_{j}\right) \text { and } \bar{x}_{i^{\prime} j}=\left(1 / n_{j}\right) \sum_{i^{\prime} \neq i^{\prime}} x_{i^{\prime} j} \text {. }
$$

While the performance of a given student (i) is affected by all other students $\left(i^{\prime} \neq i\right)$ in the $j^{\text {th }}$ class, we shall confine our graphical 
representations to a single peer (i') only, for the sake of simplicity; thus, for any given student (i), the model implied by equation 1 can be diagrammed as shown in Figure 1. Equation 1 evidently implies the direct flow of effects from (each) student (i')'s ability to student (i)'s performance, and similarly from student (i)'s ability to (each) student (i')'s performance without, in either case, letting this impact on performance be mediated by the student's own ability.

Now, it is possible to conceive of perverse situations in which one student's ability directly causes another student's performance. Honor Code scandals at West Point (or the existence of disciplinary sanctions against cheating in less prominent citadels of learning) exemplify that possibility. Once universal cheating is ruled out, however, the proponents of such a model of contextual effects must, in fact, rely on social telepathy as an intervening mechanism. Yet "action at a distance" is a well-known principle of magic, not of science which, on the contrary, is premised on the denial of that possibility and the search for intervening links.

The elevation of social telepathy to the status of an explanatory principle is by no means specific to the particular example above. Thus, let $y_{i j}$ be student aspirations rather than performance and $\mathrm{x}_{\mathrm{ij}}$ parental SES, with $\overline{\mathbf{x}}_{\mathrm{j}}$ as the school mean of parents'SES. To justify the direct effects model of equation 1 , we must again either make rather implausible assumptions about the incidence and impact of contact between each student and all other students' parents, or invoke action at a distance. Or finally, let $y_{i j}$ be student performance as before, and $\mathrm{x}_{\mathrm{ij}}$ student aspirations, with $\overline{\mathrm{x}}_{\mathrm{j}}$ the mean aspiration levels by class. Once again, we are left with the need to postulate implausible linkage mechanisms such as vicarious gratification in order to rationalize the impact of other students' aspirations on each student's performance, or we must insist on social telepathy as an explanatory principle.

Incidentally, the problem does not go away by stipulating that it is not the characteristics of all other students in the group (class or school, as the case may be) but only of those "relevant peers" which exert direct influence on one's own performance (see, e.g., Campbell and Alexander, 1965; Alexander and McDill, 1976). It 


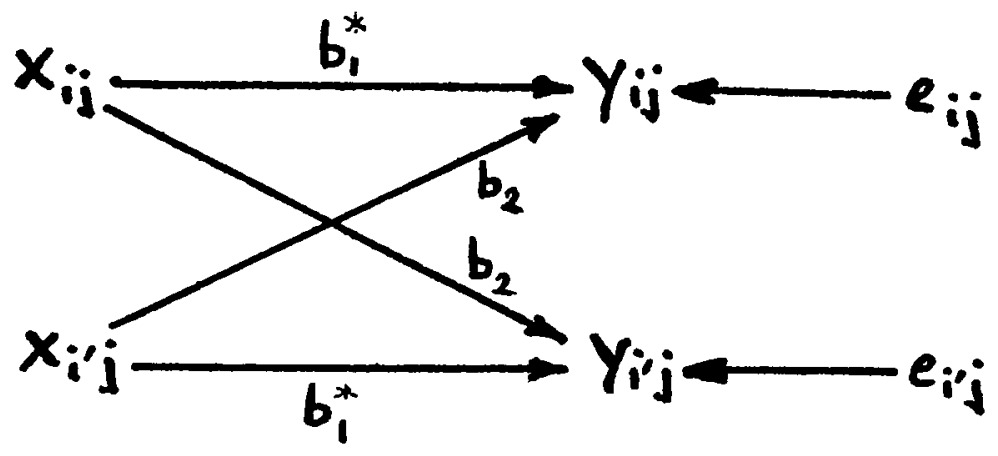

Figure 1: Social Telepathy Model of Direct Effects

is undoubtedly true that the indiscriminate inclusion of every other student's score in each student's equation (as in the case of class or school averages) is particularly unrealistic in many cases. Yet the restriction to "relevant peers" (those students with whom the individual interacts) does not eliminate the direct effects assumption of equation 1 and hence the problem of postulating implausible generating mechanisms to account for peer effects.

\section{CONVENTIONAL INTERPRETATIONS}

In considering alternative models, we direct our attention to the more plausible types of contextual mechanisms which have in fact been proposed, often in conjuction with the use of equation 1 in practice. We shall refer to them as "common fate" and "group norms," respectively, and present the corresponding structural specifications, in order to discuss their formal and statistical properties in light of the formalization of equation 1.

Common fate. Let us first consider the possibility of intervening mechanisms through which each student's performance might be at least indirectly affected by every other student's ability, as stipulated by the inclusion of group mean ability in equation 1. Thus, the average ability of students in a class might have a positive effect on the quality of instruction, e.g., by increasing pace of coverage, teacher enthusiasm, and so on. These 
global effects are presumably experienced by all students alike, as a common fate associated with membership in the same classroom.

Consequently, equation 1 should properly be replaced by the specification:

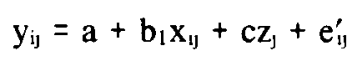

where $y_{i j}$ and $x_{i j}$ are achievement and ability as before, and $z_{j}$ is a measure of instructional treatment; $\mathbf{e}_{i j}^{\prime}$ is again an error term. Note that no $\vec{x}_{j}$ term appears in equation 5 . For any given student (i) and (each) peer (i'), the model associated with equation 5 can be diagrammed as shown in Figure 2. Of course, the common fate variable may also be defined at the school level rather than the classroom level; thus, if $y_{i j}$ is academic performance but $x_{i j}$ is parent SES, then $z_{j}$ might represent the impact of institutional resource endowments (assuming that dollars do make a difference as school administrators insist).

Clearly, if instruction quality or school resources are the postulated intervening mechanisms, direct measures of those variables should be used for $\mathrm{z}_{\mathrm{j}}$. Thus, quality of instruction could be represented in the model by measures such as amount of teacher time devoted to class preparation, amount of curricular material covered, or the like; similarly, resource endowments could be represented by a measure such as budget expenditures per student.

The implication of this type of intervening mechanism is, of course, that class average ability or school mean SES terms in equation 1 act merely as proxies for an intervening global variable such as instructional quality or school resources. Under these circumstances, any apparent "group effect" in the form of $b_{2} \overline{\mathrm{x}}_{i}^{\prime} \mathrm{j}$ in equation 4 would be indirect at best and would in fact conceal the nature of the intervening common-fate variable directly responsible for the outcome. ${ }^{1}$ Moreover, the common fate model is not necessarily consistent even with an interpretation in terms of indirect effects of $x_{i}^{\prime}$ on $y_{i j}$ mediated by $z_{j}$. For example, let $y_{i j}$ be achievement and $x_{i j}$ aspirations, but assume that $z_{j}$ represents 


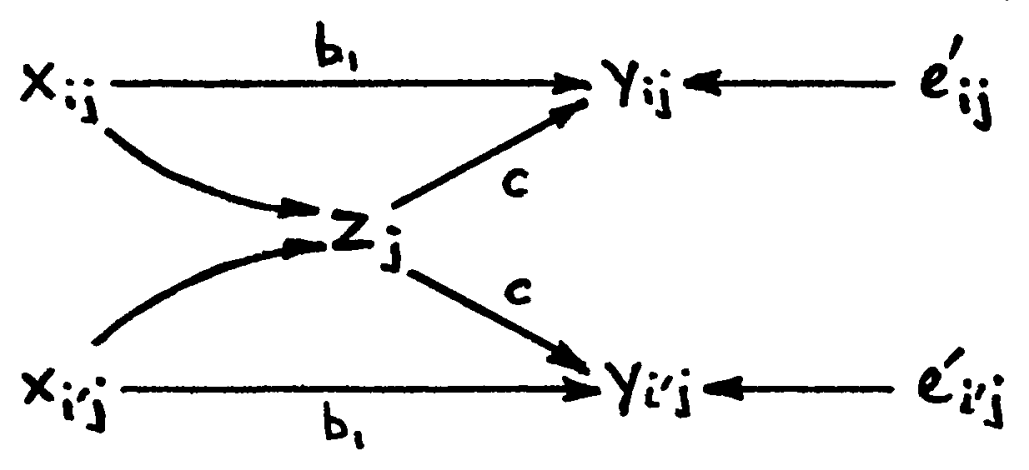

Figure 2: Common Fate Model of Indirect Effects

“exogenous" teacher characteristics (e.g., personal charisma). In that case, the common-fate variable would be a cause-rather than a consequence-of individual aspirations (as shown in Figure 3). To be sure, average aspiration level might still act as a proxy for teacher characteristics, but now the apparent effects of $\mathrm{x}_{\mathrm{i}}{ }^{\prime}$ on $\mathrm{y}_{\mathrm{ij}}$ would be spurious rather than indirect (due to their joint dependence on $z_{j}$ ).

Evidently, the common fate model does not represent a contextual process that involves the presence of others in any essential way. Rather, it remains strictly a case of individual effects, in a situation in which many individuals happen to share identical scores on a "contextual" variable, $z$, whose effects occur entirely at the individual level.

Group norms. A rather different intervening mechanism comes into view if we consider the possible role of "group norms" which are often credited as a source of contextual effects. Thus, suppose $y_{i j}$ represents achievement and $x_{i j}$ represents aspirations as before, such that the scores of all students taken together might be interpreted as defining group "norms" or "climate" with respect to educational values. Evidently, if this "climate" is to have an effect on individual performance, such an effect must be understood as the result of processes of social interaction among students. By rejecting the possibility of a direct impact of one stu- 


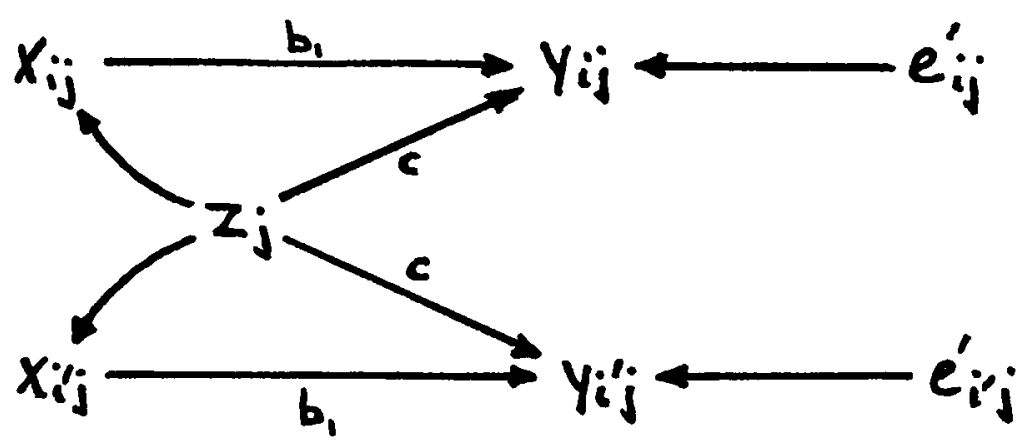

Figure 3: Common Fate Model of Spurious Effects

dent's aspirations on another's achievements as "social telepathy" we have not, of course, ruled out the possibility that such an effect could occur indirectly, via the impact of peer aspirations on one's own aspirations. Indeed the manner in which attitude and value consensus may emerge from the process of interaction among individuals suggests that the underlying model is one of mutual influence among peers, as diagrammed in Figure 4.

If the model in Figure 4 correctly represents the operation of value climates in the process of student achievement, then the corresponding specification is given by:

$$
y_{i j}=a+b_{1} x_{i j}+e_{i j}^{\prime \prime}
$$

Note that there is no direct effect of $x_{i j}{ }^{\prime}$ on $y_{i j}$; rather, the effect of $\mathrm{x}_{\mathrm{i}}{ }^{\prime}$ on $\mathrm{y}_{\mathrm{ij}}$ is mediated entirely through that of the individual determinant $x_{i j}$. Consequently, no group average term $\bar{x}_{\text {.j }}$ appears in equation 6 . In other words, one student's aspirations are assumed to influence another student's achievements only insofar as they modify the latter's aspirations. In turn the latter's aspirations generally affect performance, at the individual level, no matter what combination of background factors (including peer aspirations) may have helped to shape them.

To be sure, equation 1 misrepresents the process of Figure 4, which in reality implies a zero coefficient for the group mean 


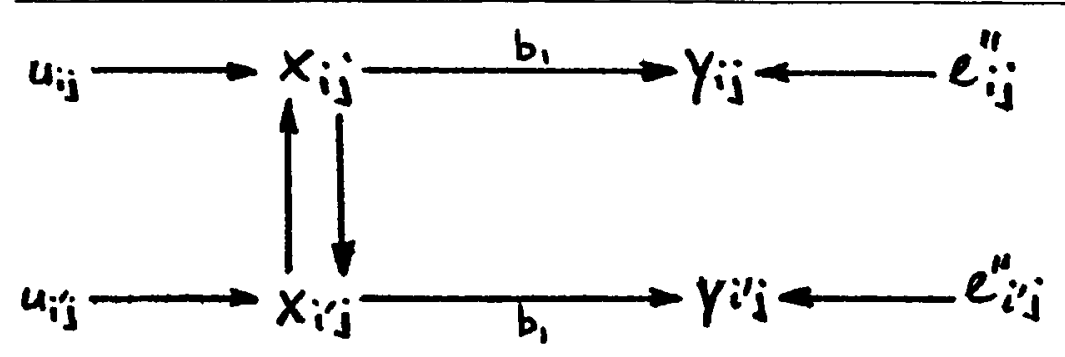

Figure 4: Social Norms Model of Indirect Effects

variable (the term $\overline{\mathbf{x}}_{i}{ }^{\prime}$ in equation 4). In practice, the implications of the structural model may easily be clouded by errors of measurement if a researcher has used imperfect indicators of the exogenous variable, $\mathrm{x}_{\mathrm{ij}}$. Suppose $\mathrm{y}_{\mathrm{ij}}$ is achievement, while $\mathrm{x}_{\mathrm{i}}^{*}$ represents the set of educational values and aspirations typically associated with family background and measured by an imperfect indicator, $x_{\mathrm{ij}}$, such as parental SES. Now, while interaction a mong students may affect student values - the "true" exogenous variable-that process will obviously not alter their parents'SES, as shown in Figure 5. Therefore, the effects of interaction on the true scores, $\mathrm{x}_{i \mathrm{j}}^{*}$, will not be reflected by the measured scores, $\mathrm{x}_{\mathrm{ij}}$.

If student values, $x_{*}^{*}$, had been measured directly, their effect on achievement would be estimated correctly by the coefficient $b *$ in Figure 5, and the effects of exogenous feedback involving the $x_{1 j}^{*}$ would remain outside the model, as in equation 6 . The consequences of using the indicator $x_{i j}$ instead are twofold. First, the impact of student values is underestimated by the coefficient $b_{1}$; second, and more importantly, the impact of other student values $x_{1 j}^{*}$ (actually zero) will be overestimated. The result will be the misleading appearance of a separate effect based on the group average $\overline{\mathbf{x}}_{i j}{ }_{j}$ of the indicator variable, as in equation 4 , since the indirect effect of other student values $\lambda_{1}^{*}$ on performance is not mediated by the measured scores, $\mathrm{x}_{\mathrm{ij} .}{ }^{2}$ Of course, the preferred strategy again is the use of a direct measure of the relevant construct, e.g., individual student values, not parent SES; alternatively, an unbiased estimate of $b_{1}$ could be obtained (e.g., via 


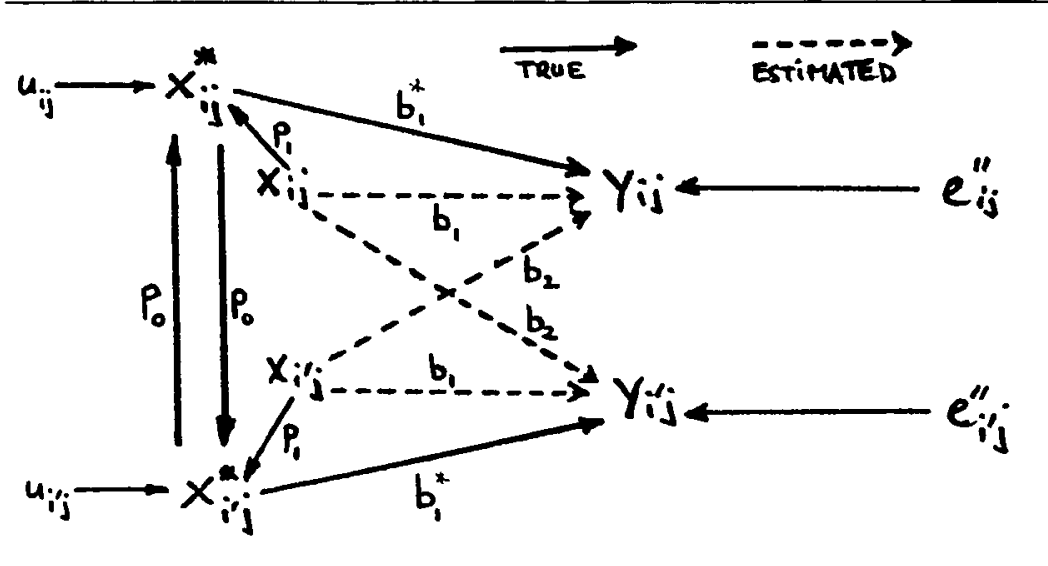

Figure 5: Social Norms Model with Measurement Error

LISREL) by using both $\mathrm{x}_{\mathrm{ij}}$ and $\overline{\mathrm{x}}_{\mathrm{i}}^{\prime}$ as fallible indicators of the unobserved variable $\mathrm{x}^{*} \mathrm{ij}$.

Unlike the intervening mechanisms considered previously, the present case-feedback among the "exogenous" variablesinvolves a genuine contextual process: interaction among individuals within a particular social structure. However, the effects of that process remain entirely hidden from view: the impact of social context on individual outcomes is confined to variables which are "exogenous" with respect to the outcome variable of interest, i.e., the impact of $x_{i j}^{\prime}$ on $x_{i j}$ cannot be represented in a model in which $y_{i j}$ is the dependent variable.

\section{SUMMARY}

The result of our examination of alternative contextual mechanisms thus far may be stated succinctly. On the one hand, we have shown that as a structural model the traditional formulation of "individual effect plus group effect" models implies rather implausible generating mechanisms for contextual effects ("social telepathy"). On the other hand, we have found that interpretrations which lead to more plausible alternative generating mechanisms are incompatible with the traditional specification, 
since the group mean terms in the conventional model of equation 1 cannot be derived from the intervening mechanisms we have considered ("common fate" or "group norms"). We do not deny the possibility that under special circumstances (e.g., measurement error or model underspecification) group averages may appear in estimation. But on substantive and logical grounds, they have no place in any of the models that result from the intervening mechanisms we have discussed thus far.

\section{ENDOGENOUS FEEDBACK}

\section{SOCIAL CONTAGION PROCESSES}

We have argued earlier that the notion of contextual effects requires explicit assumptions about, and models of, social interaction among individuals. Therefore, we now introduce an alternative intervening mechanism through which contextual effects could arise as a result of social interaction among individuals. This type of mechanism underlies such social processes as contagion, facilitation, competition, or conformity, all of which lend specific meaning to the notion of "contextual effects." What characterizes these and many other social phenomena is the idea of reciprocal influence or mutual adjustment of individuals interacting with each other. Individual behavior is assumed to be both passively responsive to the contextual cues provided by the behavior of significant others, and at the same time actively impinging upon the behavior of others sharing the same social environment.

We shall refer to the class of situations involving such processes of contextual interaction as cases of endogenous feedback, since the behavioral outcomes of interest represented by the endogenous variable are assumed to be interdependent, in addition to incorporating the effects of one or more exogenous variables. Thus suppose $y_{i j}$ is achievement and $x_{i j}$ is ability. This time, however, we assume that the performance of one student is affected by the performance (rather than a background characteristic) of 
another, and vice versa. The resulting model can be diagrammed as in Figure 6. Note that the same model would be appropriate without regard to the particular exogenous variables involved; thus in a model for student achievement, $x_{i j}$ might represent student ability, student aspirations, parent SES, or whatever. Note also that the substantive process involved (social contagion) is the same as that assumed above ("social norms"), and the model would indeed have been appropriate there if student values had been the dependent variable in the earlier case.

The formal specification corresponding to this model is characterized by two essential features. First, it includes terms in $y_{i}^{\prime} j$ in the equation for $y_{i j}$, where ( $\left.i^{\prime}\right)$ represents the relevant peers with whom (i) interacts; this has important implications for the error terms, $u_{i j}$, which are discussed more fully below. Second, the model incorporates explicit assumptions for each (i) about who the relevant peers ( $\left.i^{\prime}\right)$ are; thus the "social structure" through which the particular effects are mediated becomes an integral part of the specification of the model, as suggested by the $\mathrm{w}_{\mathrm{ii}}$, terms (and their assocated coefficient, $\alpha$ ). Since this endogenous feedback model is new to the contextual effects literature, we provide a more complete presentation of its specification and estimation below.

\section{SPECIFICATION OF THE MODEL}

We begin by considering the specification of the endogenous feedback model in Figure 6. Let us assume that the data consist of observations for all students in m classrooms, with $n_{j}$ students per class. Our contextual hypothesis states that the achievement of the $\mathrm{i}^{\text {th }}$ student in the $\mathrm{j}^{\text {th }}$ classroom, $\mathrm{y}_{\mathrm{ij}}$, is a function of his or her own academic ability, $\mathrm{x}_{\mathrm{ij}}$ and of the achievement, $\mathrm{y}_{\mathrm{i}}{ }^{\prime} \mathrm{j}$, of those other students $\left(i^{\prime}\right)$ with whom the $i^{\text {th }}$ student interacts, with con-

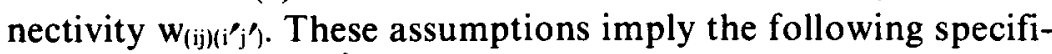
cation of the model: ${ }^{3}$ 


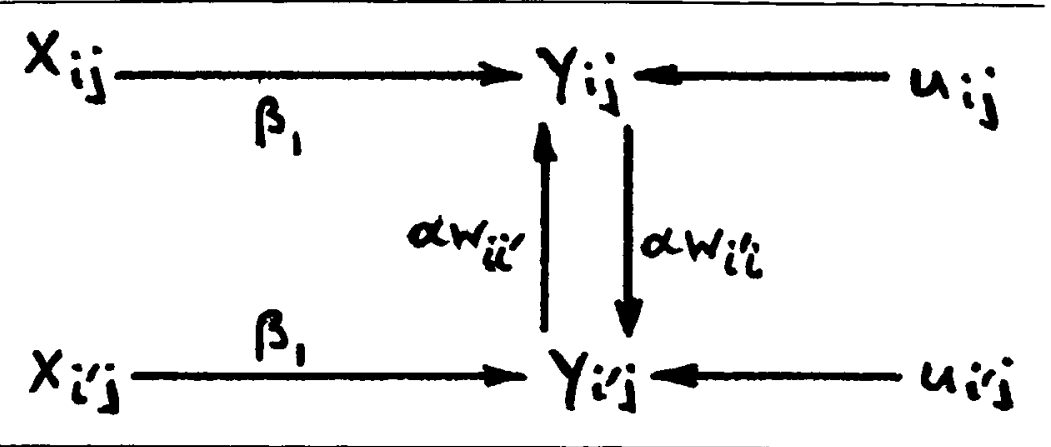

Figure 6: Endogenous Feedback Model of Social Conformity

$$
\begin{gathered}
y_{i l}=\alpha\left(\sum_{i^{\prime}=1}^{m} \sum_{i^{\prime}=1}^{n_{1}} w_{(i j)\left(i^{\prime} i^{\prime}\right)} y_{i^{\prime} i^{\prime}}\right)+\beta_{0}+\beta_{1} x_{i j}+u_{i j}, \\
\left(i=1, \ldots \ldots, n_{i} ; j=1, \ldots, m\right)
\end{gathered}
$$

Here, $a$ and $\beta_{1}$ are the regression coefficients corresponding to the terms

$$
\left(\underset{i^{\prime}=1}{m} \underset{i^{\prime}=1}{\stackrel{n}{n}} w_{(i,)\left(i^{\prime} j^{\prime},\right.} y_{i^{\prime} \prime^{\prime}}\right)
$$

and $\mathrm{x}_{\mathrm{ij}}$, respectively; $\alpha$ is a feedback coefficient representing the effect of mutual interaction among students, and $\beta_{1}$ is an impact coefficient representing the effect of a student's individual ability. $w_{(i j)\left(i^{\prime} j^{\prime}\right)}$ is a weight pertaining to the extent of interaction between student $i$ of class $j$ and student $i^{\prime}$ of class $j^{\prime}$; for two students who do not interact, $w_{(i j)\left(i^{\prime} j^{\prime}\right)}=0$, and $w_{(i j)(i j)}=0$ by definition. $u_{i j}$ is an independently distributed error term.

The general form of the model in equation 7 (assuming for convenience from here on that $n_{1}=n_{2}=\ldots=n_{j}=n$, with $m n=N$ ) is given by

$$
y=\alpha W y+X \beta+u,
$$


where:

$y$ is an $\mathrm{N}$ by 1 vector of outcome scores, $\mathrm{y}_{\mathrm{ij}}$,

$X$ is an $\mathrm{N}$ by $\mathrm{P}$ matrix of exogenous variables, which could include a constant term;

$\alpha$ is the feedback coefficient;

$\beta$ is a $P$ by 1 vector of regression coefficients for the exogenous variables;

$W$ is an $\mathrm{N}$ by $\mathrm{N}$ matrix of fixed weights representing the structure of interaction through which the $\mathrm{N}$ units of observation are connected with each other, with zeros along the main diagonal;

$u$ is an $\mathrm{N}$ by 1 vector of disturbances, $\mathrm{u}_{\mathrm{ij}}$, with $u \sim \mathrm{N}\left(0, \sigma^{2} l\right)$. ( $l$ is the identity matrix.)

The structure of the contiguity matrix, $W$, is an integral part of the model. In general, $W$ represents exogenous information about physical or social contiguity among members of the collectivity. Thus, it mirrors an essential aspect of the social structure characterizing a particular population and it defines the conduit for a variety of substantive processes which involve interaction among individuals-persuasion, contagion, competition, cooperation, emulation, and so on. Clearly, $W$ has to be specified a priori, either on the basis of data about the incidence of relevant pairwise contacts among students or, in the absence of more specific information, by resorting to simplifying assumptions about patterns of social interaction.

The definition of contiguity may vary depending on the type of process assumed to generate the contextual feedback effects. Thus, in the case of communication or contagion processes, contiguity may be based explicitly on amount of face-to-face interaction specific to each pair of students; whereas in the case of comparison or competition processes, contiguity may be defined as fixed and equal for all pairs of students in a given classroom and zero for all pairs of students from different classrooms. Moreover, the latter case of equal within-class contiguity and zero between-class contiguity may also be a plausible assumption about the probability of face-to-face interaction in the absence of further information. 
In conjunction with the appropriate contiguity matrix $W$, the feedback rate $\alpha$ is of critical importance from a substantive point of view. It provides a measure of the extent to which the outcome variable for each individual, e.g., academic performance, is affected by contiguous "spillover" from other members of the group. ${ }^{4}$ Specifically, if the rows of $W$ are normalized to sum to unity, e.g., by expressing each individual's amount of contact with others in terms of proportions, then the metric of the dependent variable is preserved by the endogenous feedback term. As a result, $\alpha$ can be interpreted as the share of individual outcomes $y_{i j}$ determined by contextual as opposed to individual mechanisms.

In its general form, the endogenous feedback model defines an entire class of processes characterized by "social contagion" phenomena. Formally, $\mathrm{y}_{\mathrm{i}}=\alpha \mathrm{y}_{\mathrm{i}}$ ' states that for $\alpha>0$ the impact of $\mathrm{y}_{\mathrm{i}}{ }^{\prime}$ on $\mathrm{y}_{\mathrm{i}}$ will be directly proportional to the score of $\mathrm{y}_{\mathrm{i}}{ }^{\prime}$, while for $\alpha<0$ the impact of $y_{i}{ }^{\prime}$ will be inversely proportional to $y_{i}^{\prime}$. Substantively the model specializes to distinct processes by virtue of alternative definitions of the sensitivity parameter $\alpha$. Thus, consensus processes (assimilation) take the form $y_{i}=\lambda\left(y_{i}^{\prime}-y_{i}\right)$ while polarization processes (contrast) take the form $y_{i}=\lambda\left(y_{i}-y_{i}^{\prime}\right)$, with $0<\lambda<1$ in both cases. In the former case, the effect of $y_{1}$ 'on $y_{i}$ is positive for $\mathrm{y}_{\mathrm{i}}<\mathrm{y}_{\mathrm{i}}{ }^{\prime}$ and negative for $\mathrm{y}_{\mathrm{i}}>\mathrm{y}_{\mathrm{i}}{ }^{\prime}\left(\mathrm{y}_{\mathrm{i}}\right.$ moves closer to $\left.\mathrm{y}_{\mathrm{i}}{ }^{\prime}\right)$; in the latter case, the opposite holds: the effect of $y_{1}^{\prime}$ on $y_{1}$ is positive if $\mathrm{y}_{1}{ }^{\prime}<\mathrm{y}_{\mathrm{i}}$ and negative if $\mathrm{y}_{\mathrm{i}}>\mathrm{y}_{\mathrm{i}}$ ( $\mathrm{y}_{1}$ moves away from $\left.\mathrm{y}_{\mathrm{i}}{ }^{\prime}\right)$. Simple algebraic manipulation then leads to the following results for

(a) assimilation:

$$
\begin{gathered}
y_{1}=\lambda\left(y_{i}{ }^{\prime}-y_{1}\right) \\
(1+\lambda) y_{1}=\lambda y_{i}{ }^{\prime} \\
y_{i}=\lambda /(\lambda+1) y_{1}{ }^{\prime}
\end{gathered}
$$

(b) contrast:

$$
y_{1}=\lambda\left(y_{i}-y_{i}{ }^{\prime}\right)
$$




$$
\begin{aligned}
& (1-\lambda) \mathrm{y}_{1}=-\lambda \mathrm{y}_{1}^{\prime} \\
& \mathrm{y}_{\mathrm{i}}=\lambda /(\lambda-1) \mathrm{y}_{\mathrm{i}^{\prime}}
\end{aligned}
$$

Thus, both cases are readily subsumed under the general form of the contagion model such that $\alpha=\lambda /(\lambda+1)>0$ represents "consensus" processes and $\alpha=\lambda /(\lambda-1)<0$ represents "polarization" processes.

In order to appreciate the role of the endogenous feedback component of the model in the contextual determination of individual outcomes, it is instructive to rearrange the terms in equation 8 to give:

$$
(I-\alpha W) y=X \beta+u
$$

Equation 9 expresses the dependent variable, net of feedback effects, as a function of the exogenous variables and the disturbances. Now, the reduced form of equation 9 is

$$
y=(I-\alpha W)^{-1} \beta+(I-\alpha W)^{-1} u
$$

Note that the inverse matrix in equation 10 is the limit of a convergent infinite series of the form

$$
(I-\alpha W)^{-1}=I+\alpha W+(\alpha W)^{2}+\ldots
$$

provided that $-1<\alpha<1$. Thus, equation 10 can be written as

$$
y=X \beta+(\alpha W) X \beta+(\alpha W)^{2} X \beta+\ldots+(I-\alpha W)^{-1} u
$$

Formally, equation 11 represents the equilibrium of the feedback structure of the model. ${ }^{5}$ Substantively, equation 11 illustrates that the final scores on the endogenous variables incorporate the results of cumulative "filtering" of the effects of exogenous variables, including their direct impact on each individual member of the group; their indirect effects due to the impact of exogenous variables on those with whom $s /$ he interacts, on those 
with whom his/her first-order interaction partners interact, and so on.

\section{ESTIMATION OF THE MODEL}

Ordinary least squares estimation of $\alpha$ and $\beta$ from equation 8 would produce inconsistent estimates of model parameters. Since a linear combination of observations for the dependent variable appears on the right hand side of equation 8 , there is feedback between the regressors and the error term in the model. This violation of the standard OLS assumptions becomes evident upon closer examination of Figure 6. The figure shows the feedback process involving the respective academic achievement levels of students in a class $\mathrm{j}$ which consists of two individuals, $i$ and $i^{\prime}$, who interact with one another with relative frequencies, $w_{\text {ii }}{ }^{\prime}$ and $w_{i^{\prime}}^{\prime}$ (and who have no significant contact outside their class). It can be seen that the impact of the performance of (i') on that of (i) is confounded with the disturbance $u_{i j}$, which becomes part of $y_{i^{\prime} j}$ by virtue of the latter's dependence on $y_{i j}$; the same holds true for the impact of the performance of (i) on that of $\left(i^{\prime}\right)$, with respect to the disturbance $u_{i}^{\prime} j$. Thus, the model is a simultaneous equation structure, and the endogenous variable must be purged of feedback effects before consistent estimates of $\alpha$ and of $\beta$ can be obtained.

In order to address the estimation problem, it is useful to proceed from the rearranged form of the basic model given by equation 9 above, which expresses the dependent variable, net of feedback effects, as a function of the exogenous variables and disturbances. If an estimate of $\alpha$ could be secured separately and if the existence of the inverse matrix $(I-\alpha W)^{-1}$ was assured, then the remaining parameters could be estimated (conditional on a fixed value of $\alpha$ ) from the reduced form in equation 10 , by applying the appropriate transformation to the exogenous variables. But the resulting estimates would still not be consistent, since the reduced-form transformation would necessarily destroy the independence property for the transformed disturbances, i.e., 


$$
\begin{aligned}
\mathrm{E}\left[(I-\alpha W)^{1} u u^{\prime}(I-\alpha W){ }^{l^{\prime}}\right] & =(I-\alpha I)^{-1} \mathrm{E}\left[u u^{\prime}\right]\left(I-\alpha W^{\prime}\right)^{1^{\prime}} \\
& =\left(\sigma^{2} I\right)\left[\left(I-\alpha W^{\prime}\right)^{\prime}(I \alpha W)\right]^{-1}
\end{aligned}
$$

which is a nondiagonal matrix.

A maximum likelihood estimation procedure that avoids the disturbance problems associated with reduced-form estimation in this case has been proposed recently by Ord (1975). The ML estimator can be derived directly from the rearranged form of the structural model in equation 9 above, i.e.,

$$
A y=X \beta+u
$$

where $A=(I-\alpha W)$.

Since this specification contains the structural disturbances, the original assumption of independently and identically distributed errors is preserved. It follows that the likelihood of $y^{\circ}=A y$ is a function of the density of $u$, where $u=A y-X \beta$. Assuming $\mathrm{f}(u) \sim \mathrm{N}\left[0, \sigma^{2} \eta\right]$, therefore,

$$
\mathrm{L}\left[y^{\circ}\right]=\mathrm{L}(A y)=\left(2 \pi \sigma^{2}\right)^{-\mathrm{N} / 2} \exp \left[-\left(1 / 2 \sigma^{2}\right)(A y-X \beta)^{\prime}(A y-X \beta)\right] .[12]
$$

The dependent variable $y^{\circ}$ is unobserved since it contains the unknown parameter $\alpha$, but the observed $y$, which do not contain the unknown parameter $\alpha$, are linear transforms of the $y$. Thus, using the Jacobian of the transformation $y^{\circ}=A y$, the resulting likelihood function of $y$ is

$$
\begin{aligned}
& \mathrm{L}[y]=\mathrm{L}\left[v^{\circ}||\left(\operatorname{Det} \frac{\partial y^{\circ}}{\partial y^{\circ}}\right)|=\mathrm{L}[\mathrm{A} y]| \operatorname{De}(A) \mid\right. \\
& =\left(2 \pi \sigma^{2}\right)^{N / 2} \exp \left[-\left(1 / 2 \sigma^{2}\right)\left(A y^{\prime} \quad X \beta\right)^{\prime}\left(A y^{\prime}-X \beta\right)\right]|\operatorname{Det}(A)|
\end{aligned}
$$

yielding the log-likelihood function

$$
\begin{aligned}
\left.\mathrm{L} *\left[\alpha, \beta, \sigma^{2} ; y\right]=(-\mathrm{N} / 2) \ln \left(2 \pi \sigma^{2}\right)-\left(1 / 2 \sigma^{2}\right) \mid(A y \quad X \beta)^{\prime}(A y-X \beta)\right] \\
+\ln |\operatorname{Det}(A)|
\end{aligned}
$$


Maximizing $L^{*}$ with respect to $\beta$ and $\sigma^{2}$ yields the maximum likelihood estimators

$$
\hat{\beta}=\left(X^{\prime} X\right)^{-1} X^{\prime} A y
$$

and, using equation 15 ,

$$
\hat{\sigma}^{2}=\left[y^{\prime} A^{\prime} M A y\right] / \mathrm{N}
$$

where

$$
M=\left(I-X\left(X^{\prime} X\right)^{-1} X^{\prime}\right)
$$

These estimators are conditional upon $\alpha$, which must be estimated by iterative grid search based on its concentrated likelihood function. The concentrated log-likelihood function for $\alpha$, using equations 15 and 16 , is given by

$$
\mathrm{L} *\left[\alpha ; y, \hat{\beta}, \hat{\sigma}^{2}\right]=[\mathrm{N} / 2(1+\ln (2 \pi))]-(\mathrm{N} / 2) \ln \left[\sigma^{2}|\operatorname{Det}(A)|^{-2 / \mathrm{N}}\right]
$$

Thus the solution is equivalent to finding the smallest value of $y^{\prime} A^{\prime} M A y /|\operatorname{Det}(A)|^{2 / \mathrm{N}}$.

The procedure suggested by Ord (1975) is based on the eigenvalues of $W$. It avoids the need to evaluate the determinant of $A=(I-\alpha W)$ afresh for each trial value of $\alpha$, and thus makes maximum likelihood estimation computationally feasible. Briefly, the eigenvalues $\lambda_{i}$ of $W$ are the roots of the polynomial

$$
|\lambda I-W|=0
$$

which can be factored into

$$
\Pi_{1}\left(\lambda-\lambda_{1}\right)=0
$$

whence

$$
|\lambda I-W|=\prod_{i}\left(\lambda-\lambda_{i}\right) .
$$


Since the eigenvalues $\lambda^{*}$ of $\alpha W$ are proportional by $\alpha$ to those of $W$, such that

$$
\left|\lambda^{*} I-\alpha W\right|=\prod_{i}\left(\lambda^{*}-\alpha \lambda_{i}\right)
$$

it follows that the required determinant is given by

$$
|I-\alpha W|=\prod_{i}\left(1-\alpha \lambda_{1}\right) \text {. }
$$

Consequently, the quantity which is to be maximized by $\hat{\alpha}$ in equation 17 is given by

$$
\operatorname{Min}[\mathbf{Q} / \mathbf{R}]
$$

where

$$
R=\left[\prod_{i}\left(1-\alpha \lambda_{1}\right)\right]^{2 / N},
$$

while

$$
\mathrm{Q}=y^{\prime} A^{\prime} M A y=y^{\prime} M y-2 \alpha y^{\prime} M W y+\alpha^{2} y^{\prime} W^{\prime} W y .
$$

Note that $Q$ is simply the error sum of squares resulting from $\hat{\beta}$ in equation 15 . The evaluation of the $\lambda_{i}$, in turn, may be further simplified, especially for large matrices, if $W$ can be forced into block-diagonal form such that eigenvalues can be obtained separately for each block. Even where this is not possible, computation may be simplified if $W$ contains a large number of zero cells as will often be the case in practice.

In sum, endogenous feedback models not only provide a theoretical specification of contextual effects; they are now also well within reach of practical estimation.

\section{SPECIFICATION BIAS}

Before concluding our discussion of estimation issues, it may be useful to consider the implications of a particular case of 
specification error which appear to be at the heart of the statistical debate over "contextual effects" associated with the traditional formulation of equation 1 . This is the case where the correct model is really the endogenous feedback model,

$$
y=\alpha W y+X \beta+u \quad[u \sim \text { IID }]
$$

but estimation is based instead on the underspecified "model"

$$
y=X \beta+v
$$

which implies that

$$
v=\alpha W y+u
$$

Now, substituting for $y$ in equation 25 reveals that the error process has the form

$$
v=\alpha W(X \beta+v)+u
$$

with the result that estimates of $\beta$ from equation 24 will be biased and inconsistent since $X$ enters into, and hence is necessarily correlated with, $v$. Moreover, equation 24 can be written as

$$
y=X \beta+[\alpha W X \beta+\alpha W v+u]
$$

with the consequence that, if (for whatever reason) a term in $W X$ were added to the underspecified "model" of equation 24 , then the resulting estimating equation

$$
y=X \beta+\alpha W X \beta+v^{*} \quad\left[v^{*}=\alpha W v+u\right]
$$

would lead to a substantively meaningless (spurious) coefficient estimate associated with $W X$ (which in any case would be a biased and inconsistent estimate of $\alpha \beta$ since, from equation $26, W X$ is correlated with the disturbance term $v^{*}$ in equation 28). Further discussion of autocorrelated error structures in contextual models is provided in the Appendix. ${ }^{6}$ 
The relationship between the conventional "group mean" formulations of "contextual effects" and the omission of underlying endogenous feedback from the model can be brought into sharper focus by considering the bias effect of that specification error in the context of OLS estimation. Obviously the effects of the specification bias in equation 24 depend on the form of $W$ (recall that there are a number of different ways to specify $W$ ). For purposes of exposition we only consider the special case in which pairs of student interactions are equally weighted within classrooms, while no significant interaction occurs across classrooms. This specification is most readily tractable and corresponds to the typical design for conventional discussions of contextual effects at the "group" level.

Thus, we assume that, for a given school, $W$ is a block-diagonal matrix containing blocks of $\left(\ell \ell^{\prime} / \mathrm{n}\right.$ ) on the diagonal (where $\ell$ is a vector of unities). ${ }^{7}$ For this definition of $W$, the basic model of equation 23 becomes

$$
y=\alpha \bar{y}+X \beta+u
$$

where

$$
\bar{y}=I \otimes\left(\ell \ell^{\prime} / \mathrm{n}\right) y=W y
$$

is a vector of group means and denotes the $\mathrm{K}$ ronecker product. Accordingly, the expectation of the OLS estimator for the incorrectly specified model in equation 24

$$
\mathrm{E}(\hat{\beta})=\beta+\mathrm{E}\left[\left(X^{\prime} X\right)^{-1} X^{\prime} \alpha W y\right]
$$

reduces to

$$
\mathrm{E}(\hat{\beta})=\beta+\mathrm{E}\left[\left(X^{\prime} X\right)^{-1} X^{\prime} \bar{y}\right] \alpha .
$$

Now, if the model without the endogenous feedback term were to be estimated from a "partitioned" (within-group) design, separately for each of the $\mathrm{j}$ classrooms, then the bias in equation 
31 would remain confined to an intercept shift (since $\bar{y}_{\cdot j}$ is constant within a given group). ${ }^{8}$ In fact even if the theoretically appropriate feedback term $\alpha \bar{y}$ were included, the vector of $\bar{y}_{. j}$ would be indistinguishable from the constant term of the model in each group, and an estimate of $\alpha$ could not be obtained (i.e., the data matrix would be singular). Thus, while the omission of endogenous feedback from the specification will result in groupspecific intercept biases, estimates of exogenous impact coefficients would remain unbiased under a "within" design.

If instead the model without the endogenous feedback term were to be estimated from a "total" (all groups combined) design, the bias in equation 31 would extend to the coefficient estimates for the exogenous variables. The reason for the latter effect becomes readily apparent by recognizing, first, that the term $\left(X^{\prime} X\right)^{-1} X^{\prime} \bar{y}$, which controls the bias in equation 31 above, is simply the regression of group means $\bar{y}_{\text {.j }}$ on the exogenous variables; and second, that this regression is necessarily nonzero whenever the regression of individual scores, $y_{1}$, on the same exogenous variables (i.e., $\left(X^{\prime} X\right)^{-1} X^{\prime} \bar{y}$ in general) is nonzero to begin with. ${ }^{9}$ Thus under a "total" analysis design the omission of endogenous feedback from the specification will lead to biased estimates of the impact of exogenous variables on individual outcomes.

There are several estimation strategies which might serve to purge coefficients for the exogenous variables of the bias resulting from omission of the endogenous feedback component. One of these would be to use a pooled design with a more complex error structure which acknowledges the existence of unspecified group-level effects, e.g., in the form of group-specific dummy variables. While this removes the bias from the coefficient estimates for the exogenous variables, it does not eliminate bias from the model altogether; it merely reallocates it to the "group" intercept terms (thus paralleling the results obtained from partitioned estimation). Furthermore, this approach precludes any possibility of distinguishing this particular source of bias from the effects of other possible specification errors, all of which are confounded by fitting group-specific intercepts. 
A second strategy which might help compensate for the omission of endogenous feedback would be to introduce the group means of the exogenous variable(s) as additional regressor(s) in the total design, as in equation 1. Recalling that the omitted feedback term, i.e., the group mean of the endogenous variable, is necessarily correlated with the group means of the exogenous variable(s) in the model, the latter in effect become proxies of the former during estimation. This estimation procedure extracts the bias from the estimated structural coefficients and converts it into coefficient estimates associated with the group means (which yield, in effect, partial intercept shifts for the groups). ${ }^{10}$ The result is essentially equivalent to the addition of group dummy variables except that it avoids the "over-fitting" of the former approach. Unlike the use of dummy variables, however, it invites substantive interpretations in terms of "group-level effects" of exogenous variables which, under the circumstances, are evidently spurious. Yet it is just this statistical relationship which appears to underlie the traditional formulation of "contextual effects" as in equation 1 .

The third strategy, and obviously the one which we would advocate, is to eliminate the bias at the outset by proper specification of the model, i.e., by allowing for the possibility of endogenous feedback. Unlike either of the other approaches, such a strategy seeks to model a social process whose validity is based on explicit substantive considerations rather than merely on statistical adjustments for group-level errors. Of course, the endogenous feedback model is not tied to the special limiting case of equal within-"group" contiguity and zero between"group" contiguity - more specific assumptions about social structure and interaction can and should be incorporated. Nor does the model depend on the particular kinds of exogenous variables involved -individual characteristics, group treatments, social background, or whatever. In any event, the question of whether a particular outcome such as student performance can legitimately (or plausibly) be associated with a specifiable process involving interaction among individuals and predicated on physical or social contiguity must be resolved on theoretical grounds. 


\section{CONCLUSION}

Our examination of possible generating mechanisms for contextual effects has led to several interesting conclusions. We find that explicit rationalizations of the conventional model of contextual effects are either formally deficient or structually misspecified. In the former case they imply the postulation of highly implausible substantive mechanisms (e.g., "social telepathy"), while in the latter case they represent the impact of variables which are independent of social interaction altogether (e.g., common fate). Moreover, among rationalizations which do acknowledge social interaction effects, those assuming feedback among exogenous variables (e.g., group norms) merely posit contextual effects beyond the reach of estimation. Only the model based on endogenous feedback effects of social interaction (i.e., social contagion) provides a specification which is both substantively meaningful and empirically compatible with contextual explanations of social phenomena.

A natural consequence of social interaction processes is interdependence of individual outcomes. While this property is theoretically attractive it is empirically troublesome since it leads to inconsistent OLS estimators of model parameters. Fortunately, recent developments of maximum likelihood estimation in spatial lattices have provided consistent estimators for endogenous feedback models. Moreover, since the same estimation problems can be shown to arise, in the form of autocorrelated errors, whenever the investigator has omitted the relevant contextual mechanisms from the specification of the model, analogous adjustments of estimation procedure are required even with underspecified models. In particular, failure to incorporate the endogenous feedback effects of social interaction in a model of individual outcomes can be shown to entail specification bias in the form of spurious estimates of conventional "group mean" effects.

These conclusions cast serious doubt on the substantive validity of contextual models based on "group mean" effects. If the notion of contextual effects is to signify substantive 
rather than statistical phenomena, then the assumption that behavior begets behavior (mediated through a given social structure) has much to recommend itself. In particular, the specification of endogenous feedback allows - indeed compelsthe investigator to make explicit the social interaction mechanisms believed responsible for "contextual effects" and the social contiguity structure which defines the relevant interaction opportunities. Where processes based on contiguity and interaction are not involved, the explanation of "group-level" effects, should they occur in estimation, must be sought elsewhere. Conversely, the endogenous feedback model avoids the need to postulate action at a distance through reified "group norms."

\section{NOTES}

1. Actually, $b_{2}$ would simply be proportional to $c_{1} \operatorname{Cov}(x, z)$.

2. In this case, the implied coefficient would be proportional to $p_{1} p_{0} b_{1}^{*}$.

3. Our model was inspired by Mitchell (1969), who uses a similar idea to treat the effects of spatial contiguity in explaining the take-over of local municipalities by guerilla insurgents in Southeast Asia. We would like to thank Howard Rosenthal for bringing this model to our attention. The model has also been discussed in Doreian and Hummon (1976: chs. 6-7).

4. The parameter $\alpha$ is analogous to the feedback rate in a dynamic model, but here feedback is filtered (weighted) by a two-dimensional structure ("lattice") of social ("spatial") contiguity rather than a one-dimensional structure of temporal contiguity.

5. Values of $\alpha$ greater than unity would imply that indirect ("filtered") effects attributable to the impact of exogenous variables on other members of the group become stronger rather than weaker with increasing "distance" (assuming that $W$ is normalized to row sums of unity). More generally, the conditions for stability or "spatial" equilibrium are that $\alpha \operatorname{Re}\left(\lambda_{1}\right)<1$, where $\operatorname{Re}\left(\lambda_{1}\right)$ is the real part of any eigenvalue $\lambda_{1}$ of $W$; thus, stability requires that $\alpha<1 / \operatorname{Re}\left(\lambda_{\max }\right)$ where $\lambda_{\max }$ is the largest eigenvalue of $W$ (assuming $\lambda_{\max }>0$ as will be the case with most definitions of contiguity). For the special case of $W$ row-normalized considered above, the largest eigenvalue is 1.0 , and hence the stability condition is: $\alpha<1$. Here, the endogenous feedback model is presented only in its equilibrium form, i.e., after all social-spatial spillover effects have run their course completely (the distribution of $y$ as mediated by the structure of the social process based on $W$ is being mapped back onto itself and is hence stable).

6. Autocorrelation is here defined in terms of a two-dimensional lattice, sometimes referred to as lattice autocorrelation or "spatial" autocorrelation.

7. Actually, $W$ should be represented by blocks of $\left(\ell \ell^{\prime}-I_{n}\right) /(n-1)$ since the main diagonal of $W$ is zero by definition: for any given individual (i), interaction effects can emanate only from (all) other members of the group (i'). Thus the conventional "group 
mean" formulation is misspecified to begin with, with the result that the parameter $\alpha$ is replaced by the quantity $\alpha[n /(n-1+\alpha)]$. However, we shall ignore this distortion for the purpose of the argument in this section.

8. This result can be derived as follows (for $W$ block-diagonal, with blocks of $W_{1}=$ $\left.\ell \ell^{\prime} / \mathrm{n}\right)$ :

Let: $X_{1}=\left[\ell \widetilde{X}_{1}\right]$, where $\ell$ is an $n$ by 1 vector of 1 's, and $\widetilde{X}_{1}$ is the $n$ by $(p-1)$ matrix of observations on the regular regressors for classroom 1; and let $y_{1}$ be the $n$ by 1 vector of observations on the dependent variable for classroom 1.

Then the "within" OLS estimator bias is given by the expectation of:

$$
\begin{aligned}
& \left(x_{1}^{\prime} x_{1}\right)^{-1} x_{1}^{\prime} \alpha w_{1} y_{1}=\alpha\left(X_{1}^{\prime} x_{1}\right)^{-1} x_{1}^{\prime} w_{1}^{\prime} y_{1} \\
& =\alpha\left[\begin{array}{ll}
\ell^{\prime} \ell & \ell^{\prime} \tilde{X}_{1} \\
\tilde{x}_{1}^{\prime} \ell & \tilde{X}_{1}^{\prime} \tilde{x}_{1}
\end{array}\right]^{-1}\left[\begin{array}{l}
\ell_{y_{1}^{\prime}} \\
\tilde{x}_{1}^{\prime}\left(\frac{\varphi^{\prime}}{\mathrm{n}}\right)_{\prime_{1}}
\end{array}\right] \\
& =\alpha\left[\left(\frac{1}{n}\right) \ell^{\prime} y_{1}^{\prime}\right]
\end{aligned}
$$

The last step uses the formula for the inverse of a partitioned matriv given in Theil (1971: 18).

9. To evtend the analysis in note 8 to a group of $m$ classrooms, we set $w^{\prime}=$ $\left(I \otimes Q Q^{\prime} / \mathrm{n}\right)$. I or this latter definition of $w$ the "total" OLS estimator bias is equivalent to the expectation of:

$$
\begin{aligned}
\left(X^{\prime} X\right)^{-1} X^{\prime} \alpha W_{y} & =\alpha\left(X^{\prime} X\right)^{-1} X^{\prime} w_{y^{\prime}} \\
& =\alpha\left[\begin{array}{ll}
\sum_{\mathrm{j}=1}^{\mathrm{m}} \ell^{\prime} \ell & \sum_{\mathrm{j}=1}^{\mathrm{m}} \ell^{\prime} \tilde{X}_{\mathrm{j}} \\
\sum_{\mathrm{j}=1}^{\mathrm{m}} \tilde{X}_{\mathrm{j}}^{\prime} \ell & \sum_{\mathrm{j}=1}^{\mathrm{m}} \tilde{X}_{\mathrm{j}}^{\prime} \tilde{X}_{\mathrm{j}}
\end{array}\right] \quad\left[\begin{array}{c}
\mathrm{n} \sum_{\mathrm{j}=1}^{\mathrm{m}} \bar{y}_{\mathrm{j}} \\
\sum_{\mathrm{j}=1}^{\mathrm{m}} \tilde{X}_{\mathrm{j}}^{\prime} \bar{y}_{\mathrm{j}}
\end{array}\right]
\end{aligned}
$$

(where $\vec{y}_{1}$ is the mean value on the dependent variable for the $\mathrm{j}^{\mathrm{t}}$ classroom, and $\tilde{X}_{1}$ is an $\mathrm{n}$ by $(P-1)$ matrix of observations for the regular exogenous variables for the $j^{\text {th }}$ classroom).

Upon expanding the expression after the last "=" sign, it will be found that for $W=$ $\left(1 \otimes \ell \ell^{\prime} / \mathrm{n}\right)$ the terms for both the constant term and the slope coefficients of the regular regressors are nonzero.

10. In effect, the inclusion of a "group mean" term when estimating an individuallevel model may serve as a practically useful device to reduce bias in the "individual 
effect" estimates under these circumstances, even though the resulting "group effect" estimates would be meaningless or inappropriate.

\section{APPENDIX}

Contextual effects may also be embedded in the error structure of a model. For example, autocorrelated errors may represent the effects of common fate variables and/or feedback processes omitted from the specification; in particular, they may arise as a result of failing to include endogenous feedback terms in the model, as discussed in the text. These implicit contextual effects can be summarized graphically, as in Figure 7. Note that the correlations among the errors could be due to common fate variables (a), feedback among "exogenous" variables (b), or endogenous feedback (c) (as indicated in Figure 7).

In these situations, the resulting autocorrelation among the error terms will require special adjustments in order to avoid inconsistent estimators. Thus, the structural specification of the "model" of Figure 7 is given by

$$
\mathrm{y}=\mathrm{X} \beta+\mathrm{u}
$$

where

$$
\mathrm{u}=\rho \mathrm{Wu}+\epsilon \quad[\epsilon \sim \mathrm{IID}]
$$

The autocorrelated errors assumption in equation A2 can be written as

$$
(\mathrm{I}-\rho \mathrm{W}) \mathrm{u}=\epsilon
$$

hence

$$
u=(I-\rho W)^{-1} \epsilon .
$$

Substituting this result back into the model (equation Al)

$$
y=X \beta+(I-\rho W)^{-1} \epsilon
$$

and multiplying through by $(\mathrm{I}-\rho \mathrm{W})$ yields

$$
(I-\rho W) y=(I-\rho W) X \beta+\epsilon .
$$




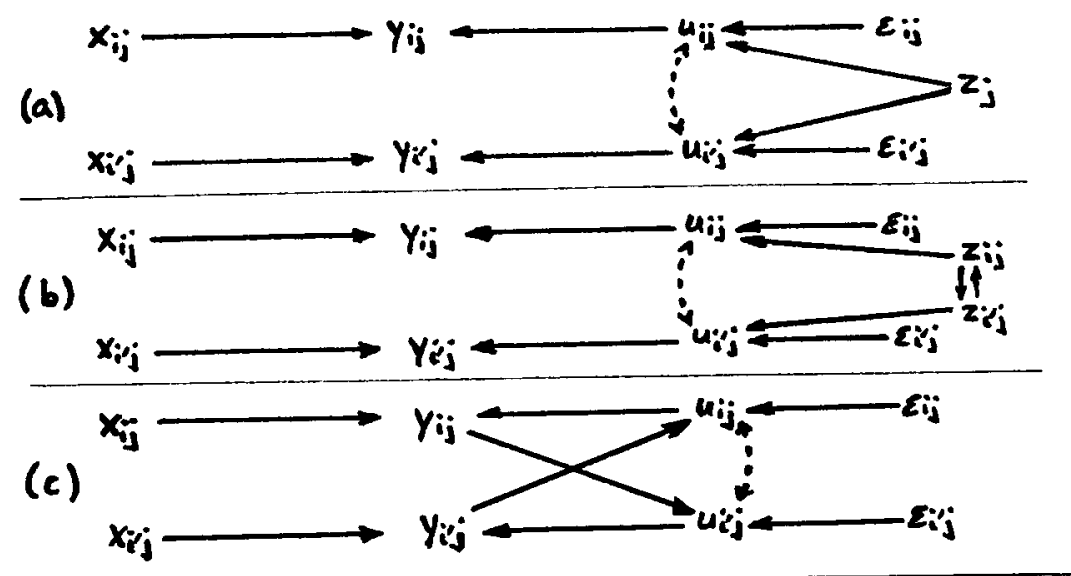

Figure 7: Implicit Context Model(s) of Correlated Errors

For $\rho$ known, equation A6 could be estimated by OLS, using

$$
Z=V \beta+\epsilon
$$

where

$$
\mathrm{Z}=(\mathrm{I}-\rho \mathrm{W}) \mathrm{y}
$$

and

$$
V=(I-\rho W) X
$$

For $\rho$ unknown, one might consider working with the formulation

$$
\mathrm{y}=\rho \mathrm{y}_{\mathrm{L}}+\mathrm{X} \beta-\mathrm{X}_{\mathrm{L}} \gamma+\epsilon
$$

where

$$
\mathrm{y}_{\mathrm{L}}=\mathrm{Wy}
$$

and

$$
X_{L}=W X
$$


subject to the nonlinear constraint

$$
\gamma=\rho \beta
$$

However, it can be shown that, even with the constraint, OLS in equation A10 will yield inconsistent estimators (analogous to the case of the endogenous feedback model considered in the text). Hence an alternative approach is needed. Ord (1975) suggests an iterative procedure, using OLS in equation A6 conditional on a given estimate of $\rho$ and using the MLE from equation 14 in equation $\mathrm{A} 3$ above on the residuals of equation $A 1$, i.e., conditional on a given estimate of $\beta$ from equation A6. The two-step process is repeated until convergence of parameter estimates is reached (Cochrane-Orcutt procedure), or until the residuals in equation A6 no longer reveal significant autocorrelation (Prais-Winston procedure), using a test proposed by Ord (1975). Optionally, iteration might be preceded by a simple grid search based on OLS in equation $A 7$ to determine an approximate starting value for $\rho$.

The explicit or implicit specification of contextual feedback effects and the methods for estimating the parameters of such models can be extended to other, more complex situations. For example, the assumption of endogenous feedback may be appropriate on theoretical grounds (social contagion process), but in addition, similar feedback processes, or common fate effects, may also be operating with respect to unspecified exogenous factors (i.e., among the error terms of the model). This situation implies an endogenous feedback model with an autoregressive error structure, i.e.,

$$
(I-\alpha W) y=X \beta+u
$$

with

$$
(\mathrm{I}-\rho \mathrm{W}) \mathrm{u}=\epsilon \quad[\epsilon \sim \mathrm{IID}]
$$

which leads to the second-order equation

$$
(\mathrm{I}-\rho \mathrm{W})(\mathrm{I}-\alpha \mathrm{W}) \mathrm{y}=(\mathrm{I}-\rho \mathrm{W}) \mathrm{X} \beta+\epsilon
$$

or

$$
\left(\mathrm{I}-(\alpha+\rho) \mathrm{W}-\alpha \rho \mathrm{W}^{2}\right) \mathrm{y}=(\mathrm{I}-\rho \mathrm{W}) \mathrm{X} \beta+\epsilon
$$


Estimation may again be accomplished by an iterative procedure, using equation A 16 to estimate $\alpha$ and $\beta$ conditional on $\rho$ by the MLE method detailed above, using equation A15 to estimate $\rho$ conditional on $\alpha$ and $\beta$, again by MLE, and repeating the process until convergent parameter estimates or uncorrelated residuals are reached. Again, a two-dimensional grid search might serve to supply starting values for $\alpha$ and $\rho$.

Comparison of equations A5 and A6 points up a further problem which will often complicate empirical analysis. Thus, equation A5 is a structural model with an infinite "moving-average" disturbance (which can be written as a first-order autoregressive error, as in equation A1), while equation A6 is merely an estimating equation which appears to suggest endogenous feedback as well as "direct" contextual effects (in the sense of social telepathy) when in fact neither assumption is part of the underlying structural model. The reverse also holds, e.g., the endogenous feedback model of the previous section

$$
(\mathrm{I}-\alpha \mathrm{W}) \mathrm{y}=\mathrm{X} \beta+\mathrm{u}
$$

in its reduced form

$$
y=(I-\alpha W)^{-1} X \beta+(I-\alpha W)^{-1} l u
$$

similarly implies an infinite "moving-average" error

$$
\mathrm{v}=(\mathrm{I}-\alpha \mathrm{W})^{-1} \mathrm{u}
$$

which, in turn, could again be written as a first-order autoregressive disturbance

$$
(\mathrm{I}-\alpha \mathrm{W}) \mathrm{v}=\mathrm{u} \text {. }
$$

Thus, except for the structure of the exogenous term, equation A6 and A18 are empirically indistinguishable, even though they are making quite different substantive assumptions about contextual processes. Finally, equation A 16 would be indistinguishable from a "genuine" second-order endogenous feedback model (to the extent that the latter assumption makes sense in a spatial or social lattice). Ultimately, as always, only prior specification on substantive grounds can guide the decision between alternative forms. 


\section{REFERENCES}

ALEXANDER, N. C. and E. L. McDILL (1976) "Selection and allocation within schools: some causes and consequences of curriculum placement." Amer. Soc. Rev. 41 (December): $963-980$.

ALWIN, D. F. (1976) "Assessing school effects: some identities." Sociology of Education 49 (October).

BARTON, A. H. (1970) "Allen Barton comments on Hauser's 'context and consex'” Amer. J. of Sociology 76 (November): 514-517.

(1968) "Bringing society back in: survey research and macro-methodology." Behavioral Scientist, 1-9.

BIDWELL, C. E. and J. D. KASARDA (1975) "School district organization and student achievement." Amer. Soc. Rev. 40 (February): 55-70.

BLAU, P. M. (1960) “Structural effects." Amer. Soc. Rev. 25 (A pril): 178-193.

BOUDON, R. (1967) L'Analyse Mathematique des Faits Sociaux. Paris: Plon.

CAMPBELL, E. Q. and C. N. ALEXANDER (1965) "Structural effects and interpersonal relationships." Amer. J. of Sociology 71 (November): 284-289.

COLEMAN, J. S. (1958) "Relational analysis: the study of social organization with survey methods." Human Organization 17: 28-36.

E. Q. CAMPBElL, C. J. HOBSON, J. McPARTLAND, A. MOOD, F. D. WEINFELD, and R. L. YORK (1966) Equality of Educational Opportunity. Washington, DC: Government Printing Office.

CRONBACH, L. J. (1976) "Research on classrooms and schools: formulation of questions, design, and analysis." Stanford, CA: Stanford Evaluation Consortium, School of Education.

DAVIS, J. A., J. L. SPAETH, and C. HUSON (1961) "A technique for analyzing the effects of group composition." Amer. Soc. Rev. 26 (A pril): 215-226.

DOREIAN, P. and N. P. HUMMON (1976) Modeling Social Processes. New York: Elsevier.

FARKAS, G. (1974) "Specification, residuals and contextual effects." Soc. Methods and Research 2 (November): 333-363.

HANNAN, M. T., J. H. FREEMAN, and J. W. MEYER (1976) "Specification of models for organizational effectiveness." Amer. Soc. Rev. 41 (February): 136-143.

HAUSER, R. M. (1974) "Contextual analysis revisited." Soc. Methods and Research 2 (February): 365-375.

-__ (1970a) "Context and consex: a cautionary tale." Amer. J. of Sociology 75 (January): 645-664.

___ (1970b) "Hauser replies." Amer. J. of Sociology 76 (November): 517-520.

LAZARSFELD, P. F. and H. MENZEL (1969) "On the relation between individual and collective properties," pp. $499-516$ in A. Etzioni (ed.) Reader on Complex Organizations. New York: Holt, Rinehart, \& Winston.

McDILL, E. L., L. C. RIGSBY, and E. D. MEYERS, Jr. (1969) "Educational climates of high schools: their effects and sources." Amer. J. of Sociology 74 (May): 567-586.

MEYER, J. W. (1970) "High school effects on college intentions." Amer. J. of Sociology 76 (July): 59-70.

MITCHELL, E. J. (1969) "Some econometrics of the Huk rebellion." Amer. Pol. Sci. Rev. 63: 1159-1171. 
ORD, K. (1975) "Estimation methods for models of spatial interaction." J. of the Amer. Statistical Association 70 (March): 120-126.

ROGOFF, N. (1961) "Local structure and educational selection," pp. 241-251 in A. H. Halsey, J. Floud, and C. A. Anderson (eds.) Education, Economy and Society. New York: Free Press.

THEIL, H. (197I) Principles of Econometrics. New York: John Wiley.

TURNER, R. H. (1964) The Social Context of Ambition. San Francisco: Chandler.

WERTS, C. E. and R. L. LINN (1971) "Considerations when making inferences within the analysis of covariance model." Educational and Psychological Measurement 31 (Summer): $407-416$.

Lutz Erbring is Assistant Professor of Political Science and Faculty Associate in the Center for Political Studies, Institute for Social Research, at the University of Michigan. In addition to quantitative models and methods of social research, his interests include mass political behavior and international relations.

Alice A. Young is Assistant Professor in the Social Science Department at Carnegie-Mellon University. Dr. Young's research interests focus on governmental regulation and organizational theory. She is currently completing two manuscripts, To Producers and Consumers of Organizations (with Lee Sproull) and Federal Regulation: An Institutional Analysis. 